\title{
The Functional Equivalence of Mental Images and Errors of Movement
}

\author{
Ronald A. Finke \\ Massachusetts Institute of Technology
}

Four experiments are reported demonstrating that mental images are functionally equivalent to physical errors of movement in producing changes in visualmotor coordination, at both central and peripheral levels of the visual-motor system. In the first experiment, subjects in one condition pointed at a target seen through laterally displacing prisms and were instructed to imagine pointing errors identical to those recorded previously for subjects in a separate condition who actually observed their pointing errors. Changes in pointing accuracy during adaptation procedures and visual-motor aftereffects following these procedures for subjects who imagined their errors were proportional to visual-motor shifts and aftereffects for subjects who observed their errors. In the second experiment, these same imagery instructions resulted in identical pointing shifts and aftereffects even in the case where prisms did not displace the target. The third experiment showed that when subjects believe that their mental images of pointing errors do not correspond to their actual pointing errors, pointing aftereffects result that are characteristic of the processing of error information at peripheral, but not central, levels of the visual motor system. The final experiment showed that when subjects do believe that their images of pointing errors correspond to actual pointing errors, but imagine the pointing movement itself in addition to their errors, pointing aftereffects result that are characteristic of the processing of error information at central, but not peripheral, levels of the visual-motor system. Contributions to visual-motor aftereffects from these two levels appear to be additive. Another significant result was that, in the imagery feedback conditions of each experiment, subjects who gave high ratings of vividness to their mental imagery showed the greatest magnitude of pointing aftereffects. These findings establish that mental images for errors of movement can produce stable visual-motor changes that cannot be accounted for simply by subjects' expectations regarding the actual consequences of their actions.

Research on human information processing has recently provided remarkable demonstrations that mental images can exhibit structural and functional characteristics that are similar to those of actual physical ob-

This research was supported by a grant from the Spencer Foundation. I am grateful to the following people, who provided many helpful comments on the project: Mary Potter, Richard Held, Stephen Kosslyn, Steve Pinker, Lynn Cooper, Peter Podgorny, Francis Ganong, David Harwood, Susan Carey, Jerry Fodor, Jerry Schneider, Bob Pachella, Jim Lackner, Gordon Bower, and Noam Chomsky. I am especially grateful to Judy Thomson, who in many ways helped the author to improve earlier versions of the manuscript. I would also like to thank Joe Bauer for technical assistance and Judy Snodgrass for preparing the figures.

Requests for reprints should be addressed to Ronald A. Finke, Department of Psychology, E10-114A, Massachusetts Institute of Technology, 79 Amherst Street, Cambridge, MA 02139. 
jects. For example, one set of studies has demonstrated that mental images can be rotated as physical objects can (Cooper \& Shepard, 1978; Shepard, 1975), while a second set of studies has demonstrated that mental images can be scanned as physical objects can (Kosslyn, 1973, 1975; Kosslyn, Ball, \& Reiser, 1978). Findings such as these are important because they constitute an empirical foundation for the study of mental imagery that is based upon quantitative experimental methods.

In addition to clarifying the nature of imagery and its relation to perception, these studies raise the very interesting question of how far this structural and functional similarity extends. Demonstrations that psychophysical judgments made on patterns that are imagined are often similar to judgments made on patterns that are observed (Posner, Boies, Eichelman, \& Taylor, 1969; Shepard \& Podgorny, 1978) suggest that images and physical objects may be functionally equivalent at specific levels of the visual system. It has been suggested that, at such levels, mental images might activate processing mechanisms in the visual system directly, substituting for or competing with external visual information (Kerst \& Howard, 1978; Moyer, Bradley, Sorensen, Whiting, \& Mansfield, 1978; Segal \& Fusella, 1970). If so, one can then raise the further question of whether mental images and physical objects might be functionally equivalent even at levels of the visual system so peripheral that the processing of visual information is known to be relatively independent of how objects are conceptualized.

Very recently, experimental techniques have been developed for finding the most peripheral level at which mental images and physical objects can have similar effects upon the visual system. One method is to have a person imagine physical objects such that information received by one who actually sees those objects is known to be processed at specific levels of the visual system, and to then test for the presence of visual aftereffects characteristic of those levels of processing. To illustrate, Finke and Schmidt $(1977,1978)$ found that mental images are functionally equivalent to physical objects at levels at which pattern information is processed but not at levels at which color information is; this finding was established by the presence or absence of orientation-specific color aftereffects. Using an alternative method, Podgorny and Shepard (1978) found that the time required to indicate whether a visual probe was located on various parts of a letter imagined on a grid was proportional to the time required when the probe was located on the corresponding parts of a letter actually present on an identical grid. The fact that these reaction times varied with probe location and letter complexity in the case of both actual and imagined letters also implied that mental images are functionally equivalent to physical objects at levels at which pattern information is processed.

The successful application of both methods to levels of pattern proces- 
sing suggests that they be applied to other visual levels, and indeed, to other visual systems. Evidence that the visual system for processing information about patterns is not identical with the system for processing information about errors of movement (designated as the visual-motor system) was reported by Schneider (1969). The studies described above suggest that images are functionally equivalent to physical objects at certain levels within the former system; perhaps it is also the case that images are functionally equivalent to physical errors of movement at certain levels within the latter system.

It is known that when errors of movement are observed, changes in visual-motor coordination result that are easily measured and which often reveal specific levels of the visual-motor system at which error information is processed (e.g., Bauer \& Held, 1975; Hay \& Pick, 1966). It is also known that at certain of these levels, visual-motor changes do not depend upon how the observed errors are conceptualized (e.g., Uhlarik, 1973; Welch, 1972). Such findings suggest that one may assess the extent to which images and physical errors of movement are functionally equivalent by comparing changes in visual-motor coordination that result when errors of movement are observed with corresponding changes that result when the same errors are imagined and movement is not observed.

A standard experimental procedure for measuring changes in visualmotor coordination resulting when movement errors are observed is that in which a person points with one hand at a visual target seen through laterally displacing prisms. When permitted to view pointing errors induced by the displacing effect of the prisms, one's accuracy improves rapidly over successive pointing trials. Removal of the prisms then results in pointing aftereffects (i.e., errors in the direction opposite the prism displacement) when further attempts are made to point at the target. Both the rate at which observed pointing errors are reduced and the extent to which pointing aftereffects for the hand observed through the prisms transfer to the hand not observed through the prisms can then be used to identify the levels of the visual-motor system at which error information is processed.'

This experimental procedure was used, with minor variations, in the present set of experiments. In the main experiment, subjects pointed at a target while unable to observe their movements and were instructed to imagine pointing errors which, on the average, would have actually occurred were they to observe their pointing hand through the displacing prisms. Measures of error reduction and pointing aftereffects for these subjects were compared with corresponding measures for a separate

\footnotetext{
${ }^{1}$ Excellent reviews of the many aspects of visual-motor adaptation to prismatic displacement may be found in Harris (1965), in Kornheiser (1976), and in Welch (1974, 1978).
} 
group of subjects who actually did observe their pointing hand through the prisms. The most peripheral level of the visual-motor system at which imagined movement errors are functionally equivalent to actual movement errors was determined by the similarity in visual-motor changes for these two groups of subjects. Three additional experiments, which were simple extensions of the main experiment, were then conducted to eliminate alternatives to mental imagery as an explanation for the resulting visual-motor aftereffects.

\section{EXPERIMENT 1}

In all experiments, subjects observed or imagined pointing errors only when their pointing movements were completed. This method was selected for the following reasons. First, the task of imagining pointing errors is greatly simplified in that subjects need to form only a single mental image corresponding to each pointing error. Secondly, previous studies have shown that pointing changes that result when subjects are merely told, upon completion of movement, the location of their unseen pointing errors are similar to those changes that result when the same errors are acturally observed (Kelso, Cook, Olson, \& Epstein, 1975; Uhlarik, 1973). While such changes may result solely from verbal descriptions of pointing errors, they could also result from mental images of pointing errors constructed spontaneously by subjects in response to these descriptions. A third reason is that pointing aftereffects in the hand observed during this adaptation procedure show approximately $60 \%$ transfer to the hand not observed (Cohen, 1967). It is very unlikely that experimental subjects could anticipate this particular percentage of intermanual transfer.

Even more important, that pointing aftereffects transfer only partially when errors are observed in this manner has been taken as evidence that error processing occurs at several distinct levels of the visual-motor system (e.g., Cohen, 1967; Welch, 1978). At levels designated as peripheral, error processing results in visual-motor changes that are rapidly established, that do not transfer to the unadapted hand, and that do not require the recognition of error with respect to an explicit visual target. At levels designated as central, error processing results in visual-motor changes that are less rapidly established, that transfer completely to the unadapted hand, and that require recognition of an explicit visual error. Since contributions to visual-motor changes from each of these levels are approximately additive (Wilkinson, 1971), resulting pointing aftereffects exhibit partial intermanual transfer. In addition, contributions from each level reach an asymptote in such a manner as to maintain this same transfer percentage except for extremely long or brief adaptation periods (Hay \& Pick, 1966; Welch, 1978). 
The relative independence of these two levels of error processing leads to the following predictions. If mental images are functionally equivalent to actual pointing errors at both central and peripheral levels of the visual-motor system, the percentage of intermanual transfer of pointing aftereffects should be the same when errors are imagined as when errors are observed, and the rates at which errors are reduced during each adaptation procedure should be proportional. By contrast, if mental images are functionally equivalent to actual pointing errors only at central levels of the visual-motor system, one should find, when errors are imagined, smaller pointing aftereffects that transfer completely to the unadapted hand and a slower rate of error reduction during adaptation procedures. Finally, if mental images are not functionally equivalent to actual pointing errors at either central or peripheral levels, one should find, when errors are imagined, no evidence for any visual-motor changes.

As a further measure, each experiment included a test for individual differences in ratings of imagery vividness. If vivid mental images of pointting errors are somehow more like observed pointing errors than are nonvivid mental images, subjects who rate their imagery as vivid ought to show greater magnitudes of pointing changes that result whenever pointing errors are imagined. Accordingly, the magnitudes of pointing changes for subjects who actually observe their errors should be greater still. That is, while the same types of visual-motor changes should be obtained in each case given that the functional equivalence extends to peripheral as well as central levels, the actual magnitudes of these changes should depend upon how closely imagined errors correspond to errors that are actually observed.

These predictions were examined in the first experiment. A noteworthy feature of this experiment is that the procedure in which pointing errors were imagined differed in three major respects from that typically used in the case where pointing errors are indicated verbally by the experimenter. First, subjects were explicitly instructed to form mental images of their pointing errors. Secondly, subjects were informed before the adaptation procedure where they were to imagine each of their pointing errors once their pointing movements were completed. A third difference was that measures were taken both of pointing changes occurring while errors were imagined and of pointing aftereffects for each hand.

Subjects

$$
\text { Method }
$$

Fifty students at MIT were paid $\$ 2.00$ for volunteer participation. All had 20-20 visual acuity or were corrected to that acuity with prescription lenses. Each subject was told that the experiment would involve pointing at a visual target, wearing a pair of goggles, and imagining various objects and events. No information was given as to the nature of the prisms that were mounted in the goggles. Informal questioning at the end of the experiment indicated that none of the subjects were aware of the purpose of the study. 


\section{General Procedure}

At the beginning of the experiment, subjects first completed the Marks (1973) Vividness of Visual Imagery Questionnaire (VVIQ). This questionnaire contains descriptions of 16 visual scenes that subjects imagine and rate for vividness on a 5-point scale. Selection of the VVIQ as the measure of imagery vividness was based on its previous success, relative to other self-reporting questionnaires, in predicting individual differences in visual memory; in particular, memory for the detailed structure and spatial configuration of visual objects (Gur \& Hilgard, 1975; Marks, 1973; White, Sheehan, \& Ashton, 1977). The subject's individual score on the VVIQ was computed at the completion of the experiment; he was later classified as either a vivid or nonvivid imager depending upon whether his score fell above or below the overall mean.

After the VVIQ was administered, the subject was fitted with the prism goggles, which were set initially so as to produce no visual displacement. Throughout the remainder of the experiment, the subject sat in front of the table illustrated in Fig. 1. An adjustable chinrest was used to accommodate differences in height, so that the subject could point comfortably underneath an occluding platform mounted on the table, which is also shown in Fig. 1. The target used for all pointing movements was a stationary red tack located directly in front of the chinrest; this target was mounted on a supporting bar whose distance from the subject could be adjusted to accommodate differences in arm length. When the region between the end of the occluding platform and the bar supporting the target was left uncovered, the subject could see his errors upon completion of his pointing movements. Alternately, when this region was covered with an opaque board, the subject could not see his errors upon completion of his pointing movements.

Each subject was told explicitly that for every pointing movement in the experiment, he was to point only to where he actually saw the target to be located. He was repeatedly reminded to do so throughout the experiment, in the attempt to discourage him from inten-

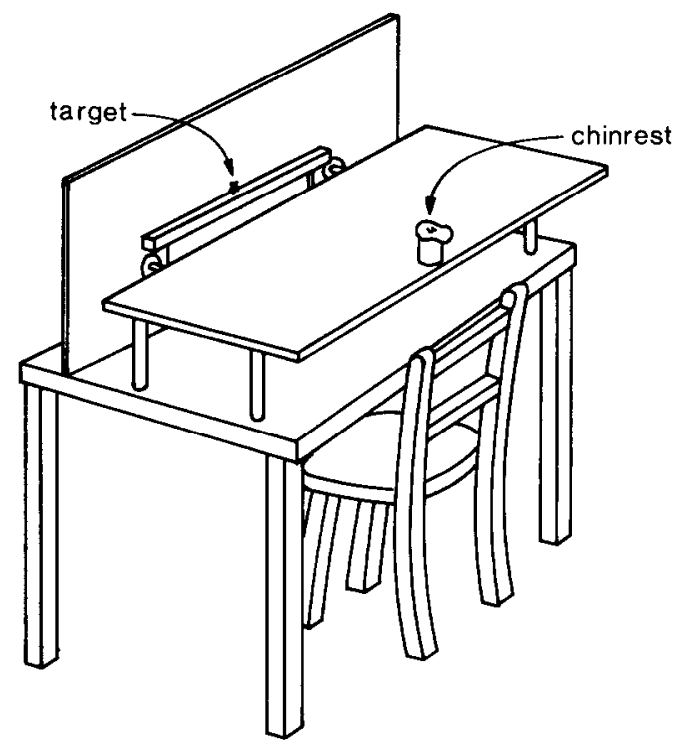

Frg. 1. The apparatus used in each experiment, showing the occluding platform and the location of the target. 
tionally pointing away from its apparent visual position. The procedure to be used for pointing at the target was then specified. The subject was asked to always point with his arm and index finger extended fully, so that his finger would arrive beyond the far edge of the supporting bar. A metronome, set at a beat frequency of one per second, was used to control the rate at which he pointed. Each movement was of 4-sec duration, and consisted of four separate 1-sec parts. The subject was instructed to point at the target on the first beat, letting his finger rest against the underside of the supporting bar; to leave his arm in its extended position on the second beat, avoiding any lateral arm movements; to withdraw his arm on the third beat; and to pause in a resting position on the fourth beat. The resting position was established by having the subject place his hands comfortably on the table, underneath the occluding platform, so that they were reasonably close to his body and separated by approximately 2 in. He was then told that this same pointing procedure was to be repeated on the following beat of the metronome, unless the experimenter indicated otherwise.

Measurements of pointing errors were taken by the experimenter during the second count of the pointing procedure; he sat at the opposite end of the table, behind the screen shown in the figure. Finger positions were recorded to the nearest $0.5 \mathrm{~cm}$, using a metric scale that was mounted on the experimenter's side of the supporting bar. At no time could the subject observe this recording procedure. As a further precaution against the subject's picking up extraneous cues regarding his actual pointing outcomes, an opaque cloth was used to cover his shoulders and upper arms.

Before actual measures were taken, the subject first practiced pointing at the target with each hand while observing his errors, until he became proficient at pointing in phase with the metronome. The feedback region was then covered, and baseline measures of pointing accuracy were recorded as the subject pointed at the target five times with his right hand, followed by five times with his left hand, without being able to observe his actual responses. For both practice and baseline pointing, the prism goggles were set so as not to displace the target.

The subject next participated in one of three adaptation conditions. In each of these conditions, the prism goggles were adjusted to produce a $6.8^{\circ}$ lateral displacement of the apparent visual location of the target. This magnitude of displacement, smaller than that of $11^{\circ}$ normally used in prism-adaptation experiments, was selected to insure that pointing errors could be imagined on both sides of the target within the same field of vision, since Kosslyn (1978) has shown that the total visual angle for imagery is approximately $20^{\circ}$. Also, subjects would be less likely to recognize a smaller prismatic displacement of the target. At no time did the experimenter disclose the nature of the effects produced by the prisms, and indicated only that a "change" was being made in the goggles as they were adjusted. The direction of displacement, to the left or to the right, was counterbalanced, and all prism adjustments were made while the subject closed his eyes.

After the prisms were adjusted for displacement, the subject opened his eyes and pointed at the target using only his right hand for 20 adaptation trials. Pointing errors for these trials were recorded and were later averaged together for groups of 5 consecutive trials. The prisms were then reset so as to produce no displacement, and baseline measures of pointing accuracy were again taken for each hand, without the subject being able to observe his pointing movements. Pointing aftereffects for each hand were then computed as the average difference between pre- and postadaptation measures of pointing accuracy.

To summarize the steps in the general procedure, the subject first practiced pointing at the target, using each hand and observing his reponses. He then pointed five times at the target using each hand without observing his responses. The prisms were then adjusted so as to displace the apparent location of the target, and the subject pointed at this apparent position using his right hand only, in one of three adaptation conditions. Finally, the prisms were reset, as before, so as to produce no displacement, and the subject again pointed five times at the target using each hand without observing his responses. 


\section{Adaptation Conditions}

Each subject participated in one of the following three adaptation conditions:

Perceptual feedback. Ten Subjects, 5 vivid and 5 nonvivid imagers, participated in this condition. These subjects were permitted to observe their pointing errors when the prisms were adjusted to displace the target.

Imagery feedback. Twenty subjects, 11 vivid and 9 nonvivid imagers, participated in this condition. These subjects were permitted to observe their pointing errors when the prisms were adjusted to displace the target. They were, however, given instructions that enabled them to imagine, on the average, the same errors that subjects in the Perceptual Feedback Condition actually did observe.

So that each subject could imagine the appropriate error on every adaptation trial, a set of visual markers was placed to one side of the target; these markers represented the average pointing errors on the initial adaptation trial and on groups of 5 consecutive adaptation trials recorded for subjects in the Perceptual Feedback Condition. The mean initial pointing error of that condition, which was found to be $4.8 \mathrm{~cm}$, was represented by a yellow tack, while tacks labcled with the numbers " 1 " to " 3 " were placed $3.7,2.4$, and $1.9 \mathrm{~cm}$ from the target, respectively, to represent the mean pointing errors on adaptation trials $1-5,6-10$, and 11-20 of that condition. ${ }^{2}$ These markers were always placed to that side of the target corresponding to the direction of actual pointing errors induced by the prisms. That is, were the subject in this condition able to see his pointing finger, he would see it arrive initially underneath the yellow tack, and would then see it gradually shift towards tack number " 3 ", as he continued to point at the apparent location of the target.

It was carefully explained to each subject that, although he was to try to point only to where he actually saw the target to be located, he was to imagine that he saw his pointing finger arrive underneath each of these markers. The order in which particular markers were to be used for this purpose was specified before he began to point at the target. The subject was told that on his very first pointing movement, he was to imagine that he saw his finger arrive underneath the yellow tack, even though he was to try to point only to where he did in fact see the target. Immediately thereafter, the experimenter would call out the number " 1 ," which meant that on his next set of pointing movements, he was to imagine that he saw his finger arrive underneath the tack labeled " 1 ," although, as before, he was to try to point only to where he saw the target. It was explained that an analogous procedure was to be followed with regard to tacks labeled " 2 " and " 3 " when the experimenter later called out these numbers, which would be called out in the same order and at the same time for all subjects. So that each subject would know precisely how to imagine his pointing errors, he was permitted to view his finger briefly under all four markers before the prisms were adjusted for displacement.

Once the prisms had been adjusted, the subject pointed at the target, and the experimenter called out the numbers 1,2 , and 3 immediately following adaptation trials 1,5 , and 10 , respectively, so that the imagined errors would correspond, on the average, to the actual errors of the previous condition. Since each subject knew where he was to imagine his pointing errors before, instead of after, he pointed at the target, he knew that his imagined error on any particular adaptation trial would not be contingent upon his actual response. Since, in addition, each subject knew that the manner in which the error markers were specified was identical for all subjects in the experiment, he also knew that his actual responses would not influence when the experimenter would indicate that a different error marker was to be used. It was not until he was debriefed at the conclusion of the experiment

${ }^{2}$ The mean pointing errors on adaptation trials $11-15$ and $16-20$ were sufficiently close together to be represented by a single marker. 
that the subject was informed that these markers referred to the positions of actual pointing errors recorded when responses could be observed. Therefore, the procedures used to specify how errors were to be imagined were such that they did not communicate to the subject where his pointing finger actually did arrive.

No feedback (Control). Twenty subjects, 9 vivid and 11 nonvivid imagers, participated in this condition. These subjects neither observed nor imagined pointing errors; rather, they were simply told to try to point accurately to where they saw the target. This condition was included to assess the extent to which uncontrolled sources of information about the erroneous outcomes of unobserved pointing movements might influence a subject's responses. For example, a subject might detect that the prisms do in fact displace the apparent position of the target, or that his pointing finger, when resting against the supporting bar, feels displaced with respect to its previously felt position. It is important to control for possible effects of these types of prism-induced cues for pointing errors, since they would also be available to subjects in the Imagery Feedback Condition.

\section{Results}

Analyses of pointing changes during adaptation procedures were performed using a 3-level Feedback Condition X 2-level Imagery Rating X 4-level Adaptation Trial design, with repeated measures on the Adaptation Trial factor. Analyses of pointing aftereffects were performed using a 3-level Feedback Condition X 2-level Imagery Rating X 2-level Pointing Hand design, with repeated measures on the Pointing Hand factor. Preliminary analyses revealed no significant effects for whether the prisms displaced the target to the left or to the right; hence, direction of displacement was not included as a separate factor.

The overall mean on the VVIQ was $2.38(N=50, S D=.65)$. For the highest scoring half of the subjects, designated as the vivid imagery group, the mean VVIQ score was $1.90(N=25, S D=.38)$. For the lowest scoring half of the subjects, designated as the nonvivid imagery group, the mean VVIQ score was $2.86(N=25, S D=.50)$.

\section{Pointing Shifts During Adaptation}

An unweighted-means analysis of variance revealed a significant main effect for Feedback Condition, $F(2,44)=6.05, p<.01$; a significant main effect for Adaptation Trial, $F(3,132)=26.31, p<.001$; and a significant Feedback Condition $X$ Adaptation Trial interaction, $F(6,132)=2.86, p<$ .05 . Figure 2 presents, for each feedback condition, the mean pointing errors across consecutive groups of five adaptation trials. For all conditions, the mean pointing error on trial 1 was $4.8 \mathrm{~cm}^{3}$.

To examine the source of the significant Feedback Condition $X$ Adaptation Trial interaction, analyses of simple main effects were conducted on these two factors, using the mixed-factor procedure indicated by Winer

\footnotetext{
${ }^{3}$ Pointing errors in each experiment are reported in centimeters of displacement. An approximate conversion to degrees of displacement may be obtained by multiplying the values in centimeters by a factor of 1.14 .
} 


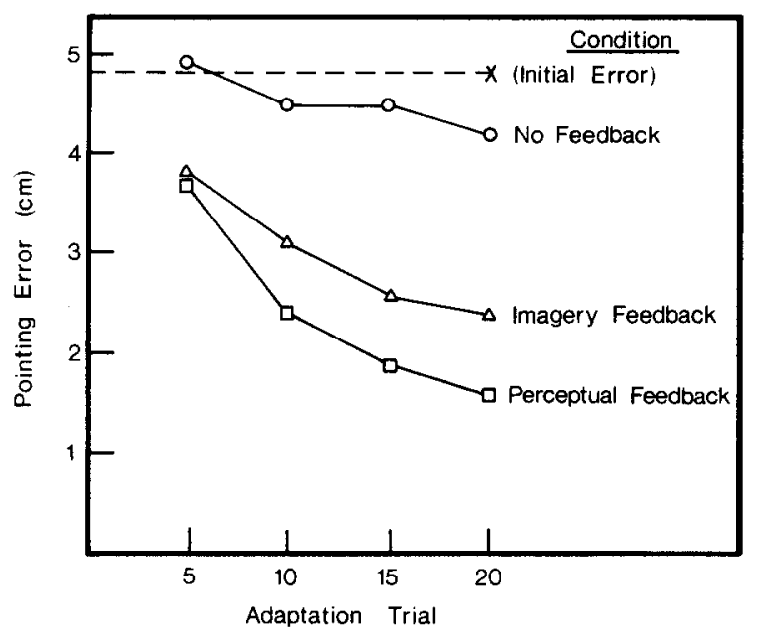

Fig. 2. Mean pointing errors across adaptation trials for feedback conditions in Experiment 1 . The mean pointing error on Trial 1 is represented by the horizontal dashed line.

(1971). A significant simple effect of Adaptation Trial was found for the Perceptual Feedback Condition, $F(3,132)=21.44, p<.001$; for the Imagery Feedback Condition, $F(3,132)=8.67, p<.001$; but not for the No Feedback Condition. A significant simple effect of Feedback Condition was found for the last three levels of the Adaptation Trial factor, $F(2,44)$ $=5.12, p<.05 ; F(2,44)=8.06, p<.01 ;$ and $F(2,44)=7.92, p<.01$, respectively. Newman-Keuls analyses of differences between means for these latter three levels revealed that while pointing errors in the No Feedback Condition were significantly greater at each level than those in both the Perceptual Feedback and the Imagery Feedback conditions, the mean pointing errors in the Imagery Feedback Condition were not significantly different at any level from those in the Perceptual Feedback Condition. The similarity between the rates of error reduction for the Perceptual Feedback and Imagery Feedback conditions is clearly shown in Fig. 2; both of these adaptation curves exhibit the characteristic negative acceleration of error reduction often reported in prism-adaptation experiments (see Welch, 1978).

At the conclusion of the experiment, subjects in the Imagery Feedback and No Feedback conditions were asked to indicate where, on the average, they thought their pointing finger actually did arrive during the adaptation procedures. Out of the $\mathbf{2 0}$ subjects in the Imagery Feedback Condition, 11 indicated that they thought their finger had arrived under the target each time, 5 indicated that they thought their finger had arrived to that side of the target corresponding to the location of their actual pointing errors, while 4 indicated that they thought their finger had arrived to that side of the target opposite their actual pointing errors. Out of the 20 
subjects in the No Feedback Condition, 15 thought their finger had arrived under the target each time, 2 thought their finger had arrived to that side of the target corresponding to their actual pointing errors, while 3 thought their finger had arrived to the opposite side of the target. Although these reports were taken informally, they suggest that those subjects who could not observe their responses were seldom aware of their actual pointing errors.

Pointing Aftereffects

An unweighted-means analysis of variance revealed significant main effects for Feedback Condition, $F(2,44)=18.09, p<.001$; Imagery Rating, $F(1,44)=5.55, p<.05$; and Pointing Hand, $F(1,44)=7.15, p<.05$; as well as a significant Feedback Condition $\mathrm{X}$ Pointing Hand interaction, $F(2,44)=3.83, p<.05$. Analyses of simple main effects on the two factors of the significant interaction revealed a significant simple effect of Feedback Condition for Right-Hand Pointing, $F(2,44)=19.90, p<.001$; and a significant simple effect of Pointing Hand for the Perceptual Feedback Condition, $F(2,44)=13.52, p<.001$. A Newman-Keuls analysis showed that the mean right-hand pointing aftereffects in the three feedback conditions were all significantly different.

The mean pointing aftereffects for all feedback conditions, imagery groups, and pointing hands are presented in Table 1. This table shows that while the mean aftereffects in the Perceptual Feedback Condition were approximately twice as large as those in the Imagery Feedback Condition, the aftereffects in these two conditions showed similar percentages of intermanual transfer. While there was also some evidence for pointing aftereffects in the No Feedback Condition, these aftereffects were of small magnitude and clearly exhibited a different type of transfer. Table 1 reveals further that vivid imagers showed greater aftereffects than nonvivid imagers in the Imagery Feedback and No Feedback conditions, but not in the Perceptual Feedback Condition. That vivid imagers showed greater aftereffects than nonvivid imagers in the No Feedback Condition was an unexpected result.

The significance of the number of subjects in each feedback condition who actually showed pointing aftereffects in the predicted directions was determined using binomial sign tests. The results of these tests, which are presented parenthetically in Table 1, reveal that all of the subjects in the Perceptual Feedback Condition and all of the vivid imagers in the Imagery Feedback Condition showed the predicted pointing aftereffects. A significant number of vivid imagers in the No Feedback Condition also showed aftereffects in the predicted direction for the average of their right- and left-hand pointing. By contrast, the number of nonvivid imagers who showed the predicted pointing aftereffects in the latter two conditions was not significant. It is of some interest to mention further that subjects in the Imagery Feedback Condition who showed relatively large pointing af- 
TABLE 1

Mean Pointing Aftereffects: Experiment 1

\begin{tabular}{|c|c|c|c|c|c|}
\hline Subject group & $N$ & $\begin{array}{l}\text { Right hand } \\
\text { (cm) }\end{array}$ & $\begin{array}{l}\text { Left hand } \\
\text { (cm) }\end{array}$ & $\begin{array}{l}\text { Average } \\
\text { (cm) }\end{array}$ & Transfer \\
\hline \multicolumn{6}{|c|}{ Perceptual Feedback Condition } \\
\hline All subjects & 10 & $\begin{array}{r}2.88 \\
\left(10^{* *}\right)\end{array}$ & $\begin{array}{r}1.41 \\
\left(10^{* *}\right)\end{array}$ & $\begin{array}{r}2.14 \\
\left(10^{* *}\right)\end{array}$ & 49 \\
\hline Vivid imagers & 5 & $\begin{array}{c}3.02 \\
\left(5^{*}\right)\end{array}$ & $\begin{array}{c}1.40 \\
\left(5^{*}\right)\end{array}$ & $\begin{array}{c}2.18 \\
\left(5^{*}\right)\end{array}$ & 46 \\
\hline Nonvivid imagers & 5 & $\begin{array}{l}2.74 \\
\left(5^{*}\right)\end{array}$ & $\begin{array}{l}1.42 \\
\left(5^{*}\right)\end{array}$ & $\begin{array}{l}2.06 \\
\left(5^{*}\right)\end{array}$ & 52 \\
\hline \multicolumn{6}{|c|}{ Imagery Feedback Condition } \\
\hline All subjects & 20 & $\begin{array}{r}1.17 \\
\left(17^{* *}\right)\end{array}$ & $\begin{array}{c}.70 \\
\left(15^{*}\right)\end{array}$ & $\frac{.94}{\left(18^{* * *}\right)}$ & 60 \\
\hline Vivid imagers & 11 & $\begin{array}{r}1.66 \\
\left(11^{* *}\right)\end{array}$ & $\begin{array}{r}1.21 \\
\left(11^{* *}\right)\end{array}$ & $\begin{array}{r}1.44 \\
\left(11^{* *}\right)\end{array}$ & 73 \\
\hline Nonvivid imagers & 9 & (6) & $(4)^{.20}$ & $(7)^{.44}$ & 29 \\
\hline \multicolumn{6}{|c|}{ No Feedback (Control) Condition } \\
\hline All subjects & 20 & $(11)^{.31}$ & $(12)^{.39}$ & $(14)^{.35}$ & 126 \\
\hline Vivid imagers & 9 & $\begin{array}{l}.51 \\
(6)\end{array}$ & $\begin{array}{l}.82 \\
(7)\end{array}$ & $\begin{array}{c}.67 \\
\left(8^{*}\right)\end{array}$ & - \\
\hline Nonvivid imagers & 11 & $(5)^{.12}$ & $\begin{array}{l}-.04 \\
(5)\end{array}$ & ${ }_{(6)}^{.04}$ & 一 \\
\hline
\end{tabular}

Note. The positive direction of pointing aftereffects is taken to be the direction opposite the prism displacement. Parentheses indicate the number of subjects in each group who actually showed the predicted aftereffects.

${ }^{a}$ Numbers in this column refer to the percentage of transfer of pointing aftereffects from the right hand to the left hand.

$$
\begin{aligned}
{ }^{*} p & <.05 \\
{ }^{* *} p & <.01 \\
{ }^{* *} p & <.001
\end{aligned}
$$

tereffects expressed genuine disbelief upon later being informed of the extent of their actual pointing changes.

\section{Discussion}

The results of Experiment 1 demonstrate that instructions to imagine errors while pointing at a prism-displaced visual target produce similar types of pointing changes as when equivalent errors are actually observed. Pointing errors observed and imagined led to proportional rates of error reduction, as well as proportional magnitudes of pointing aftereffects. As was also predicted, when errors were imagined, vivid imagers showed larger magnitudes of pointing aftereffects than nonvivid imagers. 
While these results do provide rather strong evidence that mental images are functionally equivalent to actual errors of movement at both central and peripheral levels of the visual-motor system, the finding that vivid imagers show significant pointing aftereffects in the No Feedback Condition as well must be examined more carefully. This finding implies that vivid imagers are somehow more sensitive to uncontrolled prisminduced cues that can reveal actual pointing errors. Perhaps such cues, in conjunction with instructions to imagine errors at particular locations, might have provided subjects in the Imagery Feedback Condition with sufficient information about their actual pointing responses to enable them to correct their errors without having to form mental images. It was therefore necessary to conduct a second experiment as a further control for prism-induced cues.

\section{EXPERIMENT 2}

In this experiment, the Imagery Feedback Condition of Experiment 1 was repeated using a separate group of subjects, with one modification of the adaptation procedure: at no time did the prisms actually displace the target. There are three outcomes of this procedure that would be theoretically interesting. First, if the pointing changes in the Imagery Feedback Condition of Experiment 1 resulted entirely from the availability of prism-induced cues, one should find no evidence for any pointing changes in the present experiment. Second, if imagery instructions and prisminduced cues made independent contributions to pointing changes in that condition, the magnitude of pointing changes in the present experiment should equal the difference between the magnitudes of pointing changes in the Imagery Feedback and No Feedback conditions of Experiment 1. Third, and most important, if prism-induced cues were used only when subjects could neither observe nor imagine their pointing errors, the pointing changes in the present experiment should be identical to those in the Imagery Feedback Condition of Experiment 1.

The pointing changes predicted for this latter outcome are represented in Fig. 3. Since, in the absence of prism displacement, the target is actu-

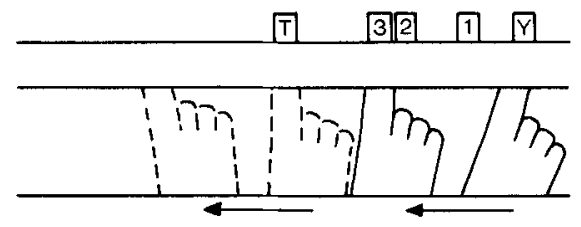

FIG. 3. Predicted pointing shifts when pointing errors are imagined. The error markers appear to the right of the target; the marker labeled " $Y$ " represents the mean pointing error on the initial adaptation trials in Experiment 1. The predicted pointing shift when errors are imagined and prisms displace the apparent position of the target to the right is indicated by the solid hands. The predicted pointing shift when the same errors are imagined and prisms do not displace the target is indicated by the dashed hands. 
ally located where it appears to be, the subject's pointing finger would initially arrive underneath the target. As he then imagined making pointing errors corresponding to markers displaced to the right of the target, his pointing finger would begin to shift, erroneously, to the left of the target. As illustrated in the figure by the dashed and solid hands, respectively, if these errors are not constrained by the same uncontrolled cues that revealed actual pointing errors in Experiment 1, the extent of pointing shift in the present experiment would be identical to that when the prisms in fact displace the target. Pointing aftereffects should also be identical with or without prismatic displacement of the target; since, presumably, a subject would continue to make the same pointing errors (i.e., to that side of the target opposite the error markers) as on his final adaptation trials.

Method

Subjects

Twenty students at MIT were paid $\$ 2.00$ for volunteer participation. None of these subjects had participated in the previous experiment or were aware of the purpose of the study.

Procedure

The procedure for the Imagery Feedback Condition of Experiment 1 was followed exactly, with the single exception that the prisms were always left so as not to displace the target. The subject was still told, as in Experiment 1, that a "change" was to be made in the goggles. When the subject closed his eyes, however, the experimenter made no changes in his adjustment of the prisms. As before, the direction of the error markers was counterbalanced.

\section{Results}

The data from the Imagery Feedback Condition of Experiment 1 were included in the present analyses, so as to determine the significance of prismatic displacement of the target when pointing errors are imagined. A Prism Condition factor was therefore established, consisting of Prism Displacement and No Prism Displacement conditions, respectively.

Analyses of pointing shifts during adaptation procedures were performed using a 2-level Prism Condition X 2-level Imagery Rating X 4-level Adaptation Trial design, with repeated measures on the Adaptation Trial factor. Analyses of pointing aftereffects were performed using a 2-level Prism Condition X 2-level Imagery Rating X 2-level Pointing Hand design, with repeated measures on the Pointing Hand factor.

The means scores on the VVIQ for subjects in Experiment 2 were essentially the same as those for subjects in Experiment 1. The overall mean VVIQ score was $2.35(N=20, S D=.49)$. For the 11 vivid imagers, the mean score was $1.99(S D=.25)$; for the 9 nonvivid imagers, the mean score was $2.79(S D=.33)$.

\section{Pointing Shifts during Adaptation}

So that the pointing shifts for the Prism Displacement and No Prism Displacement conditions could be compared directly, pointing errors for 
subjects in Experiment 2 were defined with respect to a position $4.8 \mathrm{~cm}$ from the target, on the side opposite from the error markers; that is, a distance from the target equal to the initial pointing error in Experiment 1. An unweighted-means analysis of variance revealed a highly significant effect for Adaptation Trial, $F(3,108)=13.60, p<.001$; and a significant Imagery Rating X Adaptation Trial interaction, $F(3,108)=3.78, p<.05$. Most important, however, the main effect for Prism Condition, as well as all interactions involving the Prism Condition factor, were clearly insignificant (for all analyses, $F<1$ ). The null results of these analyses showed that pointing shifts that resulted when errors were imagined and prisms displaced the target were statistically identical to pointing shifts that resulted when errors were imagined and prisms did not displace the target. The mean pointing errors across adaptation trials in the No Prism Displacement condition are presented in Fig. 5 of the following experiment; comparison of that figure with Fig. 2 shows further that virtually identical visual-motor changes were obtained in each of these conditions.

Analyses of simple main effects for the Imagery Rating X Adaptation Trial interaction revealed a single significant effect: only vivid imagers showed a significant pointing shift across adaptation trials, $F(3,108)=15.87, p<.001$. Analyzing together the data for larger numbers of subjects who imagined their pointing errors thus resolved a difference between vivid and nonvivid imagers, for this measure of pointing change, that had not been resolved in Experiment 1..

Subjects in Experiment 2 were also asked at the conclusion of the experiment to indicate where, on the average, they thought their pointing finger actually did arrive during the adaptation procedures. Out of the 20 subjects in this experiment, 9 thought their finger had arrived under the targer each time, 3 thought their finger had arrived to that side of the target corresponding to the location of their actual pointing errors, while 8 thought that their finger had arrived to that side of the target opposite their actual pointing errors (which, in the case of No Prism Displacement, was the side of the target where the error markers were located). As before, these reports suggest that subjects were not aware of their actual pointing errors.

\section{Pointing Aftereffects}

An unweighted-means analysis of variance revealed significant effects for both Imagery Rating, $F(1,36)=11.69, p<.01$; and Pointing Hand, $F(1,36)=5.05, p<.05$. As for the previous measure, the main effect for

\footnotetext{
${ }^{4}$ It could be argued that perhaps this interaction is partly due to unequal numbers of vivid and nonvivid imagers across levels of the Prism Displacement factor $(N=22,18$, respectively). Equivalent results were obtained, however, when the two lowest scoring subjects in the vivid imagery group were treated as nonvivid imagers, so as to equalize the distribution.
} 
Prism Condition, and all interactions involving the Prism Condition factor, were clearly insignificant (for all analyses, $F<1$ ). The null results of these analyses showed that pointing aftereffects for the No Prism Displacement Condition were statistically identical to those for the Prism Displacement Condition. The percentage of intermanual transfer for aftereffects in the No Prism Displacement Condition was likewise identical to that for aftereffects in the Prism Displacement Condition. The mean pointing aftereffects for the No Prism Displacement Condition are presented in Table 2 of the following experiment; comparison with Table 1 shows that virtually identical means were obtained in each case. Finally, the number of subjects in each imagery group of the present experiment who showed the predicted pointing aftereffects, presented parenthetically in Table 2, also corresponded to those for the Imagery Feedback Condition of Experiment 1.

\section{Discussion}

The results of Experiment 2 confirm the prediction that subjects who are instructed to imagine pointing errors should show identical pointing changes whether or not prisms actually displace the target. Prism-induced cues for pointing errors, while they may be used when errors cannot be observed, are evidently not used when errors can be imagined. This finding establishes further that mental images are functionally equivalent to physical errors of movement at both central and peripheral levels of the visual-motor system.

It is interesting to consider how current models of visual-motor adaptation might account for the functional equivalence at peripheral visualmotor levels. According to one model, visual-motor changes at these levels occur when observed pointing errors are compared with motor commands to the pointing arm during adaptation procedures (Hardt, Held, \& Steinbach, 1971; Held, 1965). To account for a functional equivalence between mental images and actual pointing errors at peripheral levels, this model might propose that images of pointing errors, like observed pointing errors, can be compared directly with motor commands. According to an alternative model, changes in visual-motor coordination specific to peripheral levels occur when the observed positions of the pointing arm are compared with its felt positions during adaptation procedures (Harris, 1965; Kelso et al., 1975; Mather \& Lackner, 1977). To account for a functional equivalence between mental images and actual pointing errors at peripheral levels, this model might propose that mental images of pointing errors, like observed errors, can lead to changes in how proprioceptive information is processed. Pointing changes specific to the hand for which errors are imagined could then be explained on the basis of an imagery analog to visual dominance (Posner, Nissen, \& Klein, 1976). Indeed, subjects in Experiment 2 tended to believe, erroneously, 
that their pointing finger felt to be located where they had imagined it to be.

That mental images appear to be functionally equivalent to actual errors of movement even at peripheral levels of the visual-motor system has important implications, since at those levels, visual-motor changes do not require the recognition that actual pointing errors have been made. That is, this equivalence apparently extends even to levels at which the processing of error information does not depend upon how errors are conceptualized, thus arguing that the effects of mental images for pointing errors on visual-motor coordination cannot be reduced simply to knowledge or expectations about what errors actually should occur. Moreover, having the belief that imagined errors do correspond to actual errors should be essential for visual-motor changes to occur at central levels. To claim such effects, however, also requires that they be demonstrated directly.

Since visual-motor changes occurring at these two levels are thought to be relatively independent, one should be able to selectively remove, through appropriate manipulations, the contributions from each level. The final two experiments were designed for that purpose. In Experiment 3 , an attempt was made to selectively remove visual-motor changes specific to central levels of error processing, by creating expectations that mental images of pointing errors would not correspond to actual pointing errors. Including this control is especially important since, in the previous experiments, no measures were taken to prevent subjects from regarding their imagined errors simply as the most likely consequences of any pointing changes that might be induced by the goggles. A critic could always maintain that mental images were confounded with initial expectations for actual performance.

\section{EXPERIMENT 3}

In order to separate the effects of mental images from those of expectations for performance, it is necessary to convince each subject, prior to adaptation procedures, that his actual pointing errors will not correspond to the errors he imagines. In the present experiment, a very conservative method was selected for doing so; subjects were led to believe not only that their imagined pointing errors would be false, but also that their actual pointing errors would be to the opposite side of the target from their imagined errors. This was accomplished in the following manner. Identical sets of error markers were first placed to each side of the target, as shown in Fig. 4. At the beginning of the adaptation procedure, the subject was told that a change was about to be made in his goggles, which would result in his making a specific series of pointing errors. He was told that the first time he tried to point at the target, his pointing finger would arrive underneath the yellow marker on the left side of the target; and, on later trials, underneath markers $1-3$ on the left when these numbers were 


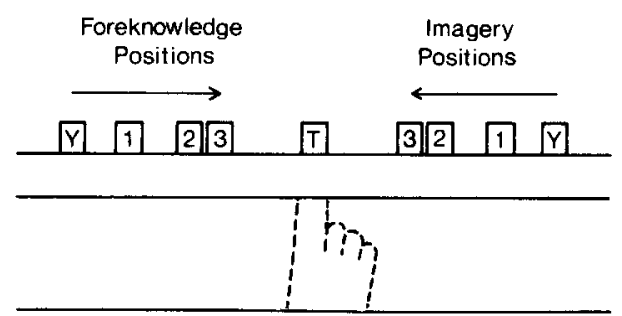

FIG. 4. Foreknowledge and imagery markers for error positions used in Experiment 3. Since the prisms did not displace the target, it was predicted that the subject's pointing finger would initially arrive underneath the target and would then shift away from the imagery markers during the adaptation procedure.

indicated by the experimenter. It was carefully explained to the subject that the markers on the left (the "foreknowledge" positions) represented the average pointing responses that had actually been made under previous conditions of the very same experiment in which he now participated. Since, as in Experiment 2, the goggles were never set to displace the target, the information the subject received about his most likely pointing outcomes was actually false.

But in addition, the subject was told that, as he pointed at the target, he was to imagine that he saw his pointing finger arrive underneath the corresponding markers on the right side of the target (the "imagery" positions). The experimenter emphasized repeatedly that the subject's pointing finger would not actually arrive at these positions; because, in fact, it would be on the other side of the target. Thus, the subject was led to expect that his imagined errors would be mirror reflections of any actual errors he was likely to make.

The following visual-motor changes were predicted as a result of this adaptation procedure. As a general prediction, if it is the case that mental images and errors of movement are functionally equivalent even at peripheral levels of the visual-motor system, mental images for pointing errors, like errors that are observed, should dominate over pointing expectations to the contrary and should determine the direction of actual pointing shifts. That is, the subject's pointing finger should shift away from the "imagery" error positions, toward the "foreknowledge" error positions.

In addition, the selective removal of central levels of error processing by this adaptation procedure should have the following specific consequences for these visual-motor changes. First, the rate at which pointing movements shift across adaptation trials should be rapid at first, due to error processing at peripheral levels, but should decrease soon thereafter without the further contribution to pointing shifts from central processing levels. Second, there should be little evidence for pointing aftereffects in the unadapted hand, while aftereffects appearing in the adapted hand 
should be reduced in magnitude by an amount equal to the usual transferred component. That is, the suppression of central levels of error processing should reduce, by a constant amount, the magnitude of pointing aftereffects in both the adapted and unadapted hands, thereby lowering the usual percentage of intermanual transfer.

\begin{abstract}
Method
Subjects

Twenty students at MIT were paid as before for volunteer participation. None of these subjects had been in previous experiments or were aware of the purpose of the present experiment.
\end{abstract}

\title{
Procedure
}

The same procedure used in Experiment 2 was used in this experiment as well, with two cxccptions: An identical set of crror markers werc placed on both sides of the target, and, as described above, the imagery instructions were preceded by information presented to the subjects that their actual pointing errors would be to that side of the target opposite their imagined errors. The direction of expected and imagined errors was counterbalanced, and, as before, subjects were told to always try to point only to where they saw the target to be located.

\section{Results}

The data for Experiments 2 and 3 were analyzed together, so as to determine the significance of expectations for pointing outcomes. It was assumed that in the previous experiment, expectations for actual pointing errors had been congruent with imagined pointing errors. Hence, a Foreknowledge Condition factor was established, consisting of Congruent Foreknowledge and Incongruent Foreknowledge conditions, respectively. Analyses of pointing shifts during adaptation procedures were performed using a 2-level Foreknowledge Condition X 2-level Imagery Rating $X$ 4-level Adaptation Trial design, with repeated measures on the Adaptation Trial factor. Analyses of pointing aftereffects were performed using a 2-level Foreknowledge Condition X 2-level Imagery Rating X 2level Pointing Hand design, with repeated measures on the Pointing Hand factor.

The results for the VVIQ were similar to those obtained previously. For subjects in the present experiment, the mean VVIQ score was $2.34(N=20$, $S D=.41)$. For the 12 vivid imagers, the mean score was $2.10(S D=.29)$; for the 8 nonvivid imagers, the mean score was $2.70(S D=.29)$.

\section{Pointing Shifts During Adaptation}

For all analyses, pointing errors were defined exactly as in Experiment 2; that is, with respect to the imagery error markers. An unweightedmeans analysis of variance revealed a significant Foreknowledge Condition X Adaptation Trial interaction, $F(3,108)=3.41, p<.05$. The basis for this interaction is illustrated by Fig. 5 , which presents the mean point- 


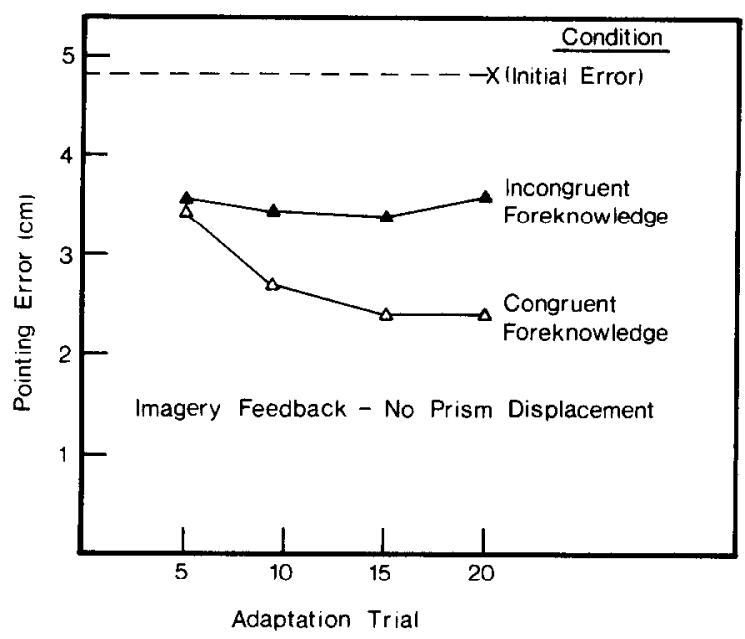

Fig. 5. Mean pointing shifts across adaptation trials for Experiments 2 and 3 . In each experiment, errors of movement were imagined, and the prisms did not displace the target. The Congruent Foreknowledge Condition is the same condition as the No Prism Displacement Condition, as labeled in Experiment 2.

ing shifts for the Congruent Foreknowledge and Incongruent Foreknowledge conditions. As is shown in this figure, through the first five adaptation trials, rapid pointing shifts away from the imagery error positions occurred in both conditions. On subsequent adaptation trials, however, no further pointing shift occurred in the Incongruent Foreknowledge Condition, while in the Congruent Foreknowledge Condition, pointing movements continued to shift away from the imagery positions. The additional visual-motor shift on later adaptation trials, absent in the Incongruent Foreknowledge Condition, was one predicted effect of error processing specific to central levels.

As in the previous experiments, subjects in the present experiment were asked to give postexperimental reports regarding their perception of where their pointing finger had arrived during the adaptation procedures. When these subjects were asked to indicate where, on the average, their pointing finger had actually felt to be located when resting against the supporting bar, nine indicated that their finger felt like it had arrived under the target each time, four indicated that their finger felt like it had arrived to that side of the target where the foreknowledge markers were located, while seven indicated that their finger felt like it had arrived to that side of the target where the imagery markers were located. Moreover, each of these seven subjects believed that the purpose of the study had been to see if the pointing finger would shift from the foreknowledge markers all the way to the imagery markers. Thus, subjects in this experiment tended 
to misperceive both the location of their pointing errors and the actual direction of their pointing shifts.

\section{Pointing Aftereffects}

An unweighted-means analysis of variance revealed significant main effects for Foreknowledge Condition, $F(1,36)=5.98, p<.05$; and Pointing Hand, $F(1,36)=4.02, p<.05$; while the main effect for Imagery Rating approached significance in the predicted direction $(p<.10)$. The mean pointing aftereffects for each condition, imagery group, and pointing hand are presented in Table 2. This table shows, first of all, that the direction of these aftereffects, like that of pointing shifts during adaptation procedures, was determined not by the expected errors, but rather by the imagined errors. Aftereffects in the Incongruent Foreknowledge Condition were smaller than those in the Congruent Foreknowledge Condition, but as predicted, this difference consisted of an equal reduction in the magnitudes of aftereffects for each pointing hand. As a comparison of

TABLE 2

Mean Pointing Aftereffects: Experiments 2 and 3

\begin{tabular}{|c|c|c|c|c|c|}
\hline Subject group & $N$ & $\begin{array}{l}\text { Right hand } \\
\text { (cm) }\end{array}$ & $\begin{array}{l}\text { Left hand } \\
\text { (cm) }\end{array}$ & $\begin{array}{l}\text { Average } \\
\text { (cm) }\end{array}$ & Transfer $^{a}$ \\
\hline \multicolumn{6}{|c|}{ Congruent Foreknowledge Condition: Experiment 2} \\
\hline All subjects & 20 & $\begin{array}{r}1.34 \\
\left(17^{* *}\right)\end{array}$ & $\begin{array}{r}.81 \\
\left(17^{* *}\right)\end{array}$ & $\begin{array}{c}1.07 \\
\left(18^{* * *}\right)\end{array}$ & 60 \\
\hline Vivid imagers & 11 & $\begin{array}{c}1.72 \\
\left(9^{*}\right)\end{array}$ & $\begin{array}{r}1.11 \\
\left(11^{* *}\right)\end{array}$ & $\begin{array}{c}1.41 \\
\left(11^{* *}\right)\end{array}$ & 65 \\
\hline Nonvivid imagers & 9 & $\begin{array}{c}.96 \\
\left(8^{*}\right)\end{array}$ & (6) & $(7)^{.73}$ & 53 \\
\hline \multicolumn{6}{|c|}{ Incongruent Foreknowledge Condition: Experiment 3} \\
\hline All subjects & 20 & $\begin{array}{r}.60 \\
\left(16^{* *}\right)\end{array}$ & ${ }_{(10)^{.15}}$ & $\begin{array}{r}.38 \\
\left(1 \kappa^{* *}\right)\end{array}$ & 25 \\
\hline Vivid imagers & 12 & $\begin{array}{c}.97 \\
\left(10^{*}\right)\end{array}$ & ${ }^{.12}$ & $\begin{array}{r}.55 \\
\left(11^{* *}\right)\end{array}$ & 12 \\
\hline Nonvivid imagers & 8 & (6) & ${ }_{(5)}^{.19}$ & (5) & - \\
\hline
\end{tabular}

Note. Subjects in each condition imagined pointing errors, and the prisms never displaced the target. The positive direction of pointing aftereffects is taken to be the direction opposite the imagined errors. The Congruent Foreknowledge Condition is the same condition as the No Prism Displacement Condition, as labeled in Experiment 2. Parentheses indicate the number of subjects in each group who actually showed the predicted aftereffects.

${ }^{a}$ Numbers in this column refer to the percentage of transfer of pointing aftereffects from the right hand to the left hand.

$$
\begin{aligned}
{ }^{*} p & <.05 \\
{ }^{* *} p & <.01 \\
* * * & <.001
\end{aligned}
$$


the mean pointing aftereffects for all subjects in these two conditions reveals, central levels of error processing, present in the case of congruent foreknowledge, contributed approximately $0.7 \mathrm{~cm}$ of error to the aftereffects of both the adapted and unadapted pointing hands. Accordingly, aftereffects in the Incongruent Foreknowledge Condition exhibited a lower percentage of intermanual transfer. This reduction of intermanual transfer was most evident for vivid imagers and was also indicated by the number of subjects who actually showed the predicted pointing aftereffects in each hand, presented parenthetically in Table 2.

\section{Discussion}

The results of the present experiment support the predicted consequences of selectively removing error processing at central levels of the visual-motor system. Most important, mental images for pointing errors, even when contrary to expectations for actual pointing errors, still determine the direction of pointing shifts and pointing aftereffects. Apparently, mental images of pointing errors, like observed pointing errors, can dominate pointing shifts at levels of the visual-motor system at which the processing of error information does not depend upon how the errors are conceptualized. This finding provides yet further evidence that mental images are functionally equivalent to actual errors of movement even at peripheral visual-motor levels.

That foreknowledge about actual pointing errors incongruent with imagined errors would selectively reduce pointing changes specific to error processing at central levels was also supported by the results of this experiment. First, when imagined errors are incongruent with expected errors, changes in visual motor coordination occur only during the initial adaptation trials. The resulting adaptation function is characteristic of early contributions from peripheral levels of error processing; contributions from central levels, absent in this case, are more important during later adaptation trials (Hay \& Pick, 1966; Welch, 1978). Second, this adaptation procedure results in the reduction of that component of pointing aftereffects that is exhibited equally by either pointing hand, thought to be due to central levels of error processing (Cohen, 1967). Moreover, it was not the case that the aftereffects in this experiment were smaller simply because less adaptation as a whole was produced, since the percentage of intermanual transfer was clearly lowered as well, especially for vivid imagers.

The results of this experiment therefore demonstrate that when imagined errors are regarded as false, pointing changes characteristic of error processing at central levels of the visual-motor system can be selectively removed. The final experiment was designed to show that, under other conditions, pointing changes characteristic of error processing at peripheral levels of the visual-motor system can likewise be selectively removed. 


\section{EXPERIMENT 4}

As discussed previously, pointing changes specific to peripheral levels of error processing are thought to result either from a comparison between errors of movement and motor commands to the pointing arm or from a comparison between errors of movement and the felt position of the pointing arm. Both comparisons necessarily require that pointing movements actually be performed when errors are imagined. Thus, if subjects were to imagine the pointing movement itself during adaptation procedures in addition to pointing errors, pointing changes specific to peripheral levels of error processing should be selectively removed. Accordingly, if subjects also were led to belicve that their imagined crrors would correspond to actual errors were the pointing movements to be performed, pointing changes should result that are characteristic of error processing occurring at central levels only. Under these conditions, equivalent pointing aftereffects should appear in each pointing hand, equal in magnitude to the usual transferred component. That is, one should find smaller pointing aftereffects that show a larger percentage of intermanual transfer.

If error processing at central and peripheral levels does in fact make independent contributions to visual-motor changes, one should also find that the aftereffects in the present experiment (which, presumably, would be due exclusively to central processing levels), when added to the aftereffects in the previous experiment (which, presumably, were due exclusively to peripheral processing levels), should be equal to the aftereffects in Experiment 2 (which, presumably, were due both to central and peripheral processing levels). This prediction follows from numerous demonstrations, in the case of perceptual feedback, that contributions from these two levels are additive (Hay \& Pick, 1966; Wilkinson, 1971).

Method

\section{Subjects}

Twenty students at MIT were selected and paid as in the previous experiments. None of these subjects were aware of the purpose of the present experiment.

\section{Procedure}

The procedure of Experiment 2 was again used, with the following variation. Before instructions were given for imagining pointing errors in the adaptation procedure, the subject was first asked to practice imagining the pointing movement itself, without observing or imagining his errors, until he could do so in phase with the metronome. Ten practice trials were usually sufficient for this purpose; on occasion, when the subject experienced some difficulty in performing the mental pointing movements, 10 additional practice trials were included. He was then instructed that, although he was to continue to imagine trying to pointing accurately only to where he saw the target, he was also to imagine that he saw his pointing finger arrive under the designated error marker on the second count of the pointing procedure. To simplify this task, the experimenter identified the beat on which the subiect was to imagine that each pointing movement had been initiated, and specified, as in previous experiments, which error markers were to be used. It was carefully explained to the subject 
that the order in which these markers were to be specified corresponded to where his finger actually would arrive, were he really to point at the target and were he able to observe his responses.

In all other respects, the experiment was conducted exactly as Experiment 2 . The subject was told that a change was to be made in his goggles, although the prisms never displaced the target. As before, the direction of error markers was counterbalanced.

\section{Results}

Scores on the VVIQ were similar to previous scores. The overall mean on the VVIQ was $2.50(N=20, S D=.62)$. For the 9 vivid imagers, the mean score was $1.97(S D=.24)$; for the 11 nonvivid imagers, the mean score was $2.94(S D=.47)$.

Analyses of pointing aftereffects were performed using a 2-level Imagery Rating X 2-level Pointing Hand design, with repeated measures on the Pointing Hand factor. An unweighted-means analysis of variance clearly showed no evidence either for a main effect for Pointing Hand $(F<1)$ or for an Imagery Rating $X$ Pointing Hand interaction $(F<1)$. The main effect for Imagery Rating, while failing to reach significance, approached significance in the predicted direction $(p<.10)$. The mean pointing aftereffects, presented in Table 3, were in the same direction as in the previous experiments, away from the imagery markers. As predicted, similar magnitudes of pointing aftereffects were obtained for each pointing hand, indicating that error processing had been restricted to central visualmotor levels. The larger percentage of intermanual transfer, most evident for vivid imagers, was also revealed by the number of subjects who actually did show the predicted aftereffects, presented parenthetically in the table.

TABLE 3

Mean Pointing Aftereffects: Experiment 4

\begin{tabular}{|c|c|c|c|c|c|}
\hline Subject group & $N$ & $\begin{array}{l}\text { Right hand } \\
\text { (cm) }\end{array}$ & $\begin{array}{l}\text { Left hand } \\
\quad(\mathrm{cm})\end{array}$ & $\begin{array}{c}\text { Average } \\
\text { (cm) }\end{array}$ & Transfer $^{a}$ \\
\hline All subjects & 20 & ${ }_{(13)}^{.68}$ & ${ }_{(13)^{.58}}$ & $\begin{array}{r}.63 \\
\left(16^{* *}\right)\end{array}$ & 85 \\
\hline Vivid imagers & 9 & $\begin{array}{l}.90 \\
(7)\end{array}$ & $\begin{array}{c}1.00 \\
\left(8^{*}\right)\end{array}$ & $\begin{array}{r}.95 \\
\left(9^{* *}\right)\end{array}$ & 111 \\
\hline Nonvivid imagers & 11 & ${ }_{(6)}^{.45}$ & $(5)^{.15}$ & ${ }_{(7)}^{.30}$ & - \\
\hline
\end{tabular}

Note. Subjects in this experiment imagined pointing movements in addition to errors during the adaptation procedure, and the prisms never displaced the target. The positive direction of pointing aftereffects is taken to be the direction opposite the imagined errors. Parentheses indicate the number of subjects in each group who actually showed the predicted aftereffects.

${ }^{a}$ Numbers in this column refer to the percentage of transfer of pointing aftereffects from the right hand to the left hand.

$$
\begin{aligned}
{ }^{*} p & <.05 \\
{ }^{* *} p & <.01
\end{aligned}
$$


TABLE 4

Effects of Foreknowledge and Movement on Mean Pointing Aftereffects: Imagery Feedback without Prism Displacement

\begin{tabular}{|c|c|c|c|c|c|c|}
\hline (E) & Foreknowledge & Movement & $N$ & $\begin{array}{l}\text { Right hand } \\
\text { (cm) }\end{array}$ & $\begin{array}{l}\text { Left hand } \\
\text { (cm) }\end{array}$ & Transfer \\
\hline \multicolumn{7}{|c|}{ All subjects } \\
\hline (2) & Congruent & Real & 20 & 1.34 & .81 & 60 \\
\hline (3) & Incongruent & Rcal & 20 & .60 & .15 & 25 \\
\hline (4) & Congruent & Imagined & 20 & .68 & .58 & 85 \\
\hline \multicolumn{3}{|c|}{ Sum of (3) and (4) } & & 1.28 & .73 & 57 \\
\hline \multicolumn{7}{|c|}{ Vivid imagers } \\
\hline (2) & Congruent & Real & 11 & 1.72 & 1.11 & 65 \\
\hline (3) & Incongruent & Real & 12 & .97 & .12 & 12 \\
\hline (4) & Congruent & Imagined & 9 & .90 & 1.00 & 111 \\
\hline \multicolumn{3}{|c|}{ Sum of (3) and (4) } & & 1.87 & 1.12 & 60 \\
\hline \multicolumn{7}{|c|}{ Nonvivid imagers } \\
\hline (2) & Congruent & Real & 9 & .96 & .51 & 53 \\
\hline (3) & Incongruent & Real & 8 & .22 & .19 & - \\
\hline (4) & Congruent & Imagined & 11 & .45 & .15 & - \\
\hline \multicolumn{4}{|c|}{ Sum of (3) and (4) } & .67 & .34 & 51 \\
\hline
\end{tabular}

Note. The conditions listed are those for Experiments 2, 3, and 4, respectively, as denoted by the numbers in parentheses.

The prediction of additivity for contributions from each level is examined in Table 4, which summarizes the effects of whether foreknowledge is congruent or incongruent with imagined errors and whether movement itself is imagined or actually performed. In support of this prediction, the aftereffects for each pointing hand in Experiments 3 and 4 are approximately additive with respect to the corresponding aftereffects in Experiment 2. This independence of central and peripheral levels was revealed most clearly by the vivid imagers, who showed the largest difference in intermanual transfer between the last two experiments.

\section{Discussion}

Whereas the results of the previous experiment showed that pointing aftereffects specific to central levels of error processing could be selectively removed, leaving effects due to error processing at peripheral levels only, the results of the present experiment showed that aftereffects specific to peripheral levels could be selectively removed as well, leaving effects due to error processing at central levels only. Since these contributions to visual-motor aftereffects appear to be additive, Experiments 3 and 4 show directly that mental images can be functionally equivalent to 
actual errors of movement at central levels independently from peripheral levels and at peripheral levels independently from central levels. As with Experiment 3, it was not simply the case that less overall adaptation had been produced in the present experiment, for while the aftereffects were again smaller than in Experiment 2, the percentage of intermanual transfer was greater. The present experiment also speaks directly to the criticism that expectations for actual pointing errors could have been totally responsible for the results of the earlier experiments, since, without the accompanying presence of physical movement, these expectations could have accounted for no more than the functional equivalence at central visual-motor levels.

\section{GENERAL DISCUSSION}

The major findings of this study may be summarized as follows. Instructions to imagine pointing errors were found to result in pointing changes proportional to those that result when identical errors are observed. These pointing changes were characteristic of the processing of error information at both central and peripheral levels of the visual-motor system. Those pointing changes characteristic of the processing of error information at peripheral levels were present when subjects were led to expect that their actual pointing errors would not correspond to their imagined pointing errors, but they were not present when acts of movement as well as errors of movement were wholly imaginary. The converse resulted in the case of central levels. Those pointing changes characteristic of the processing of error information at central levels were present when acts of movement as well as errors of movement were wholly imaginary, but they were not present when subjects were led to expect that their actual pointing errors would not correspond to their imagined pointing errors. For all cases in which pointing errors were imagined, subjects rating their imagery as vivid showed greater pointing shifts and aftereffects. I claim that these findings demonstrate that mental images are functionally equivalent to actual errors of movement at the lowest level of the visual-motor system revealed by measurable changes in visual-motor coordination.

These findings have several important implications for the empirical investigation of mental imagery. First, since experimental subjects as a rule do not know precisely the nature of pointing changes that result when their movements are observed through prisms, it is unlikely that they could intentionally give responses to conform with those predicted in this study. That subjects could intentionally give responses to conform with responses predicted is an objection that has often been made of current work on imagery. It is difficult, however, to conceive how subjects in this study could have anticipated the predicted percentages of intermanual transfer of pointing aftereffects or the predicted rates of 
visual-motor shifts in any of the experimental conditions. Even if they were to know precisely each of the predicted responses, they could not have used their knowledge to monitor the extent of their actual pointing errors, since they could not observe their pointing movements during testing procedures. I therefore suggest, as a methodological proposal, that measures of mental imagery should be made on subjects who are not aware of their actual responses.

Secondly, the method used in this study has the further advantage that measures of visual-motor aftereffects follow imagery-inducing procedures. Since these measures are taken after subjects no longer imagine pointing errors, they reveal the extent to which mental images can have residual effects upon the visual-motor system. Previous research has seldom explored the possibility that changes in visual systems caused by mental images may persist even after images are no longer present.

Even more important, the results of this study demonstrate that mental images can have residual effects on visual-motor coordination that are not simply a consequence of how images are conceptualized. This finding is of particular theoretical interest, since, at the present time, there is much controversy about whether images are stored spatially or propositionally (Anderson, 1978; Kosslyn \& Pomerantz, 1977). While the results of this study do not resolve that issue, they do suggest important constraints upon the range of effects that either model of imagery could explain. For example, one wonders whether both models could provide equally satisfying accounts of the fact that visual motor aftereffects result even in the case where knowledge about actual errors is contrary to imagined errors. Perhaps the identification of specific anatomical structures that are involved at each level of visual-motor processing could help to answer this question, by placing neurological limitations on the types of representational formats in which images could be stored.

As a further issue, while imagery and perceptual feedback produced the same types of visual-motor changes, the result that larger aftereffects were obtained with perceptual feedback merits additional consideration. In order to account for this difference, one must identify which feedback characteristics, common to imagery and perception, determine the size of visual-motor aftereffects. For example, do mental images of pointing errors result in smaller aftereffects because they correspond to observed pointing errors that are more difficult to resolve, or perhaps, that are less stable? Similar questions could be raised with regard to the result that vivid imagers showed larger aftereffects than nonvivid imagers in the imagery feedback conditions, Knowing precisely what the VVIQ measures when subjects rate their imagery would help, but unfortunately, performance measures that reliably correlate with the VVIQ do not suggest an answer. However, one might systematically degrade perceptual feedback along relevant dimensions, and attempt to match the smaller 
aftereffects that would result with those aftereffects resulting in the case of imagery feedback. Proportional changes in the size of aftereffects should then be found when vivid imagers degrade imagery feedback along the same dimensions.

I have so far discussed the implications of these findings for theories about the nature of imagery. They also have practical implications as well. Previous work has suggested that the actual performance of movement can often be facilitated by imagining the performance of movement (Stuart \& Richardson, 1964). The results of this study suggest that people who need to practice their motor skills would do best not to imagine the entire performance, but rather to actually perform, imagining how it looks. Their visual-motor coordination would be maximally influenced were they to imagine their movement errors in conjuction with actual movement and were they to expect that their imagined errors would correspond to their actual errors.

Finally, it would be interesting to repeat the present experiments, inviting subjects to imagine the locations of the error markers in addition to inviting them to imagine their pointing errors. Recent work on scanning mental images suggests that subjects can imagine accurately the threedimensional locations of physical objects (Pinker \& Kosslyn, 1978). In light of that work, it is reasonable to expect that subjects could carry out the quite complicated task which repeating the present experiments in that way would require of them. One could then determine whether appropriate errors could be imagined in the total absence of any visual cues. This was not done in the present study, however, because I felt it to be essential to maintain precise control over where subjects imagined each of their pointing errors.

\section{REFERENCES}

Anderson, J. R. Arguments concerning representations for mental imagery. Psychological Review, 1978, 85, 249-277.

Bauer, J., \& Held, R. Comparison of visually guided reaching in normal and deprived infant monkeys. Journal of Experimental Psychology: Animal Behavior Processes, 1975, 1, $298-308$.

Cohen, M. M. Continuous vs. terminal visual feedback in prism aftereffects. Perceptual and Motor Skills, 1967, 24, 1295-1302.

Cooper, L. A., \& Shepard, R. N. Transformations on representations of objects in space. In E. C. Carterette \& M. Friedman (Eds.), Handbook of perception New York: Academic Press, 1978. Vol. 8.

Finke, R. A., \& Schmidt, M. J. Orientation-specific color aftereffects following imagination. Journal of Experimental Psychology: Human Perception and Performance, 1977, 3, 599-606.

Finke, R. A., \& Schmidt, M. J. The quantitative measure of pattern representation in images using orientation-specific color aftereffects. Perception \& Psychophysics, 1978, 23, 515-520.

Gur, R. C., \& Hilgard, E. R. Visual imagery and the discrimination of differences between 
altered pictures simultaneously and successively presented. British Journal of Psychology, 1975, 66, 341-45.

Hardt, M. E., Held, R., \& Steinbach, M. J. Adaptation to displaced vision: A change in the central control of sensorimotor coordination. Journal of Experimental Psychology, $1971,89,229-239$.

Harris, C. S. Perceptual adaptation to inverted, reversed, and displaced vision. Psychological Review, 1965, 72, 419-444.

Hay, J. C., \& Pick, H. L. Visual and proprioceptive adaptation to optical displacement of the visual stimulus. Journal of Experimental Psychology, 1966, 71, 150-158.

Held, R. Plasticity in sensori-motor systems. Scientific American, 1965, 213, 84-94.

Kelso, J. A. S., Cook, E., Olson, M. E., \& Epstein, W. Allocation of attention and the locus of adaptation to displaced vision. Journal of Experimental Psychology: Human Perception and Performance. 1975, 1, 237-245.

Kerst, S. M., \& Howard, J. H. Memory psychophysics for visual area and length. Memory \& Cognition, 1978, 6, 327-335.

Kornheiser, A. Adaptation to laterally displaced vision: A review. Psychological Bulletin, $1976,83,783-816$.

Kosslyn, S. M. Scanning visual images: Some structural implications. Perception \& Psychophysics, 1973, 14, 90-94.

Kosslyn, S. M. Information representation in visual images. Cognitive Psychology, 1975, 7, 341-370.

Kosslyn, S. M. Measuring the visual angle of the mind's eye. Cognitive Psychology, 1978, $10,356-389$.

Kosslyn, S. M., Ball, T. M., \& Reiser, B. J. Visual images preserve metric spatial information: Evidence from studies of imagery scanning. Journal of Experimental Psychol. ogy: Human Perception and Performance, 1978, 4, 47-60.

Kosslyn, S. M., \& Pomerantz, J. R. Imagery, propositions, and the form of internal representations. Cognitive Psychology, 1977, 9, 52-76.

Marks, D. F. Visual imagery differences in the recall of pictures. British Journal of Psychology, 1973, 64, 17-24.

Mather, J. A., \& Lackner, J. R. Adaptation to visual rearrangement: Role of sensory discordance. Quarterly Journal of Experimental Psychology, 1977, 29, 237-244.

Moyer, R. S., Bradley, D. R., Sorensen, M. H., Whiting, J. C., \& Manfield, D. P. Psychophysical functions for perceived and remembered size. Science, 1978, 200, 330-332.

Pinker, S., \& Kosslyn, S. M. The representation and manipulation of three-dimensional space in mental images. Journal of Mental Imagery, 1978, 2, 69-84.

Podgorny, P., \& Shepard, R. N. Functional representations common to visual perception and imagination. Journal of Experimental Psychology: Human Perception and Performance, 1978, 4, 21-35.

Posner, M. I., Boies, S. J., Eichelman, W. H., \& Taylor, R. L. Retention of visual and name codes of single letters. Journal of Experimental Psychology Monograph, 1969, 79, (1, Pt. 2).

Posner, M. I., Nissen, M. J., \& Klein, R. M. Visual dominance: An information-processing account of its origins and significance. Psychological Review, 1976, 83, 157-171.

Schneider, G. E. Two visual systems. Science, 1969, 163, 895-902.

Segal, S. J., \& Fusella, V. Influence of imaged pictures and sounds on detection of visual and auditory signals. Journal of Experimental Psychology, 1970, 83, 458-464.

Shepard, R. N. Form, formation, and the transformation of internal representations. In $\mathbf{R}$. L. Solso (Ed.), Information processing and cognition: The Loyola symposium. Hillsdale, NJ: Erlbaum, 1975.

Shepard, R. N., \& Podgorny, P. Cognitive processes that resemble perceptual processes. In 
W. K. Estes (Ed.), Handbook of learning and cognitive processes. Hillsdale, NJ: Erlbaum, 1975.

Start, K. B., \& Richardson, A. Imagery and mental practice. British Journal of Educational Psychology, 1964, 34, 280-284.

Uhlarik, J. J. Role of cognitive factors on adaptation to prismatic displacement. Journal of Experimental Psychology, 1973, 98, 223-232.

Welch, R. B. The effect of experienced limb identity upon adaptation to simulated displacement of the visual field. Perception \& Psychophysics, 1972, 12, 453-456.

Welch, R. B. Research on adaptation to rearranged vision: 1966-1974. Perception, 1974, 3, 367-392.

Welch, R. B. Perceptual modification: Adapting to altered sensory environments. New York: Academic Press, 1978.

White, K., Sheehan, P. W., \& Ashton, R. Imagery assessment: A survey of self-report measures. Journal of Mental Imagery, 1977, 1, 145-170.

Wilkinson, D. A. Visual-motor control loop: A linear system? Journal of Experimental Psychology, 1971, 89, 250-257.

Winer, B. J. Statistical principles in experimental design. New York: McGraw-Hill, 1971.

(Accepted December 4, 1978) 